\title{
HST observations of the nebula around the central compact object in the Vela Jr. supernova remnant ${ }^{\star}$ (Research Note)
}

\author{
R. P. Mignani ${ }^{1}$, A. De Luca $^{2,3}$, and A. Pellizzoni ${ }^{4}$ \\ 1 Mullard Space Science Laboratory, University College London, Holmbury St. Mary, Dorking, Surrey, RH5 6NT, UK \\ e-mail: rm2@mssl.ucl.ac.uk \\ 2 Istituto Universitario di Studi Superiori Viale Lungo Ticino Sforza 56, Pavia 27100, Italy \\ 3 INAF, Istituto di Astrofisica Spaziale, via Bassini 15, Milan 20133, Italy \\ e-mail: deluca@iasf-milano.inaf.it \\ 4 INAF, Osservatorio Astronomico di Cagliari, Localitá Poggio dei Pini, Strada 54, Capoterra, Cagliari 09012, Italy \\ e-mail: apellizz@oa-cagliari.inaf.it
}

Received 30 July 2009 / Accepted 13 October 2009

ABSTRACT

\begin{abstract}
Context. A handful of young (a few thousand years) supernova remnants (SNRs) host point-like X-ray sources, dubbed central compact objects (CCOs), which are thought to be radio-silent isolated neutron stars formed by the supernova explosion. So far, no $\mathrm{CCO}$ has been firmly detected at other wavelengths. However, ground-based observation in the $\mathrm{H}_{\alpha}$ band detected a nebula around CXO J085201.4-461753, the CCO in the Vela Jr. SNR. The nebula has also been detected in deep $R$-band observations performed with the Very Large Telescope (VLT). Interestingly, both its extension and its flux in the $R$ band are consistent with those measured in $\mathrm{H}_{\alpha}$, suggesting that the nebula spectrum is dominated by line emission, possibly produced by a velocity-driven bow-shock in the interstellar medium (ISM) or by its photo-ionisation from the neutron star.

Aims. The aim of this work is to resolve the morphology of the $\mathrm{H}_{\alpha}$ nebula around the CCO to verify the proposed interpretations. Methods. We performed high-resolution imaging observations of the nebula with the Wide Field Planetary Camera 2 (WFPC2) of the Hubble Space Telescope (HST) through the $656 \mathrm{~N}$ filter, almost exactly centred on the rest wavelength of the $\mathrm{H}_{\alpha}$ line.

Results. Surprisingly enough, we did not detect the nebula in our WFPC2 image down to a $3 \sigma$ flux limit of $\sim 3 \times 10^{-15} \mathrm{erg} \mathrm{cm}^{-2} \mathrm{~s}^{-1}$. This limit is a factor of 10 fainter than the nebula flux measured in the discovery ground-based observations which were, however, performed with redder and broader $\mathrm{H}_{\alpha}$ filters.

Conclusions. The non-detection of the nebula in the narrower and bluer WFPC2 $656 \mathrm{~N}$ filter suggests that the peak of the emission might actually be at longer wavelengths. One possibility, compatible with the bow-shock scenario only, is that the $\mathrm{H}_{\alpha}$ line is redshifted by $\sim 10-60 \AA$ due to the neutron star motion with a radial velocity $450 \lesssim V_{\mathrm{r}} \lesssim 2700 \mathrm{~km} \mathrm{~s}^{-1}$. The other possibility is that the nebula is a knot of [NII] emission $(\lambda=6583.6 \AA)$ unrelated to CXO J085201.4-461753 and possibly associated with the nearby star Wray 16-30. High-resolution spectroscopy observations will help to settle the nature of the nebula.
\end{abstract}

Key words. stars: neutron - ISM: supernova remnants - ISM: clouds

\section{Introduction}

$\mathrm{X}$-ray observations performed over the last 30 years led to the discovery of about ten puzzling radio-silent, isolated neutron stars (INSs) at the centre of young $(\sim 0.3-20$ kyears old) supernova remnants (SNRs), hence dubbed central compact objects (CCOs; Pavlov et al. 2004). Their thermal-like X-ray spectra and lack of pulsar-wind nebulae (PWNe) indicate a low level of magnetospheric activity, which clearly sets CCO apart from most young INSs (see De Luca 2008 for a recent review). X-ray pulsations have been measured only for 1E 1207-5209 in PKS 1209-52 $(P=424 \mathrm{~ms}$; Zavlin et al. 2000), CXOU J185238.6+004020 in Kes $79(P=105 \mathrm{~ms}$; Gotthelf et al. 2005), and RXJ0822.0-4300 in Puppis A ( $P=112 \mathrm{~ms}$; Gotthelf \& Halpern 2009). In all three cases, the upper limits on the period derivatives (Gotthelf \& Halpern 2007;

* Based on observations with the NASA/ESA Hubble Space Telescope, obtained at the Space Telescope Science Institute, which is operated by AURA, Inc. under contract No NAS 5-26555.
Halpern et al. 2007; Gotthelf \& Halpern 2009) suggest magnetic fields $B \lesssim 10^{11-12} \mathrm{G}$, rotational energy losses $\dot{E} \lesssim 10^{32-35} \mathrm{erg} \mathrm{s}^{-1}$, and spin-down ages a factor of $\gtrsim 1000$ larger then the SNR age. This suggests a scenario where CCOs are born spinning close to their present period and with very low magnetic fields, perhaps enabling polar cap accretion from a debris disc. So far, no CCO has been detected outside the X-ray band (see De Luca 2008, and references therein). One possible exception is the CCO in the RXJ 0852.0-4622 (G266.1-1.2) SNR. In the optical, CXO J085201.4-461753 is embedded in a compact ( $\sim 6^{\prime \prime}$ diameter) nebula detected in $\mathrm{H}_{\alpha}$ both in an archival ESO/MPG $2.2 \mathrm{~m}$ observation and in a digitised UK Schmidt Telescope (UKST) plate (Pellizzoni et al. 2002). Recent Very Large Telescope (VLT) observations clearly detected the nebula also in the $R$ band (Mignani et al. 2007a,b) with an extension and a flux consistent with those measured in $\mathrm{H}_{\alpha}$, suggesting that its spectrum is dominated by line emission. The most likely interpretations favour either a velocity-driven bow-shock nebula, 
as observed in a handful of other INSs (see, e.g. Pellizzoni et al. 2002, and references therein), or a photo-ionisation nebula produced by the ultraviolet (UV) radiation from the neutron star. The nebula was undetected in more recent VLT infrared (IR) observations which, instead, pinpointed a faint $\left(K_{\mathrm{S}} \sim 21.4\right)$ candidate counterpart to the CCO (Mignani et al. 2007b).

Here, we present high resolution $\mathrm{H}_{\alpha}$ imaging observations of the nebula around CXO J085201.4-461753 taken with the Hubble Space Telescope (HST). The observations and data analysis are described in Sect. 2, while the results are discussed in Sect. 3.

\section{Observations and results}

As a back-up after the failure of the Advanced Camera for Survey (ACS) on January 2007, CXO J085201.4-461753 was re-scheduled for observations with the Wide Field Planetary Camera 2 (WFPC2). We obtained a set of four $500 \mathrm{~s}$ exposures during the allocated spacecraft orbit on January 12th 2008. In order to exploit the maximum spatial resolution of the WFPC2 we centred the target in the planetary camera $(P C)$ chip ( $0{ }^{\prime} 045 /$ pixel $)$. The spacecraft pointing and orientation were fine-tuned in order to place two bright and nearby field stars, HD 76060 ( $V=7.9$; $\approx 35^{\prime \prime}$ northeast) and Wray 16-30 ( $V=$ $13.8 ; \approx 25^{\prime \prime}$ southwest), out of the $P C$ field of view. The observations were performed using the $656 \mathrm{~N}$ filter which has a central wavelength $\lambda=6564 \AA$ and a full width half maximum (FWHM) $\Delta \lambda=22 \AA$. We retrieved the data from the HST science data archive after on-the-fly re-calibration, performed to remove instrument signatures (bias and flat-field correction). Flux calibration was performed as a part of the WFPC2 standard pipeline processing. Single exposures were finally combined and averaged using the STSDAS task combine to filter cosmic ray hits.

As a reference for the CXO J085201.4-461753 position we used the Chandra coordinates re-computed in Mignani et al. (2007b), i.e. $\alpha(\mathrm{J} 2000)=08^{\mathrm{h}} 52^{\mathrm{m}} 01.37^{\mathrm{s}}, \delta(\mathrm{J} 2000)=$ $-46^{\circ} 17^{\prime} 53^{\prime \prime} .50$, with a nominal $90 \%$ error circle of $0{ }^{\prime} .6$. For consistency with Mignani et al. (2007b), we checked the astrometric calibration of the WFPC2 image against the positions of stars in the 2MASS catalogue (Skrutskie et al. 2006). We used the mosaic of the four WFPC2 chips since it provides a large enough field of view to include a sufficient number of $2 M A S S$ stars. We produced the mosaic image with the STSDAS task wmosaic which also accounts for the correction of the geometric distortions of the four chips. Then, we measured the pixel coordinates of the $2 M A S S$ stars through gaussian fitting with the Graphical Astronomy and Image Analysis (GAIA) tool ${ }^{1}$ and we computed the pixel-to-sky coordinates transformation using the code ASTROM ${ }^{2}$. This yielded an rms $\sigma r \approx 0.13$ in the astrometric fit. Finally, we added in quadrature the uncertainty in the registration of the WFPC2 image on the 2MASS reference frame $\left(\sigma_{\mathrm{tr}}=0 .^{\prime} 055\right)^{3}$. Thus, the overall accuracy of the WFPC2 astrometry is 0 . $^{\prime} 14(1 \sigma)$. By adding in quadrature this value to the 0 '. 6 accuracy of the Chandra coordinates we obtained an uncertainty of $\sim 0$.' 62 on the CXO J085201.4-461753 position on the WFPC2 image.

No point or extended source is visible at or close to the CCO position (Fig. 1). In order to increase the signal-to-noise ratio we rebinned the image by a factor of 2 and applied a smoothing

\footnotetext{
1 star-www.dur.ac.uk/ pdraper/gaia/gaia.html

2 http://star-www.rl.ac.uk/Software/software.htm

3 As in Lattanzi et al. (1997), $\sigma_{\mathrm{tr}}=\sqrt{3} \times \sigma_{\mathrm{s}} / \sqrt{N}_{\mathrm{s}}$, where $N_{\mathrm{s}}$ is the number of $2 M A S S$ stars and $\sigma_{\mathrm{s}}=0.2$ is their conservative mean positional error (Skrutskie et al. 2006).
}

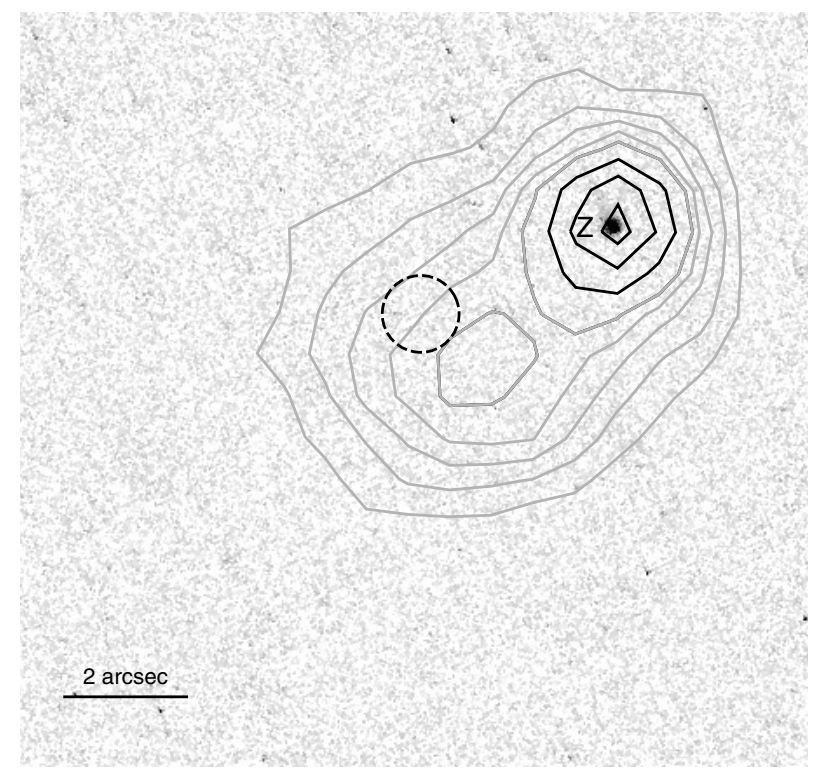

Fig. 1. $13^{\prime \prime} \times 13^{\prime \prime}$ part of the combined $2000 \mathrm{~s}$ WFPC2 image of the CXO J085201.4-461753 field obtained through the $656 \mathrm{~N}$ filter. The circle ( 0.62 radius) corresponds to the computed uncertainty of the $\mathrm{CCO}$ position on the WFPC2 (see Sect. 3). Star Z of Pavlov et al. (2001) is labelled. North to the top, East to the left. The isophotal contours from the $U K S T \mathrm{H}_{\alpha}$ image of Pellizzoni et al. (2002) overplotted (light grey contours: linear spacing; dark grey contours: logarithmic spacing).

over $3 \times 3$ pixel cells. Still, our result did not change. We thus conclude that the CXO J085201.4-461753 nebula was not detected in our images. We evaluated the upper limit to the flux of the undetected nebula from the number of background counts over regions with a size comparable to that of the nebula. We thus estimated that a $3 \sigma$ detection would correspond to a count rate of $0.92 \mathrm{cts} \mathrm{s}^{-1}$ over such an area. According to the WFPC2 absolute flux calibration, this corresponds to a flux limit of $\sim 3 \times 10^{-15} \mathrm{erg} \mathrm{cm}^{-2} \mathrm{~s}^{-1}$. Assuming pure $\mathrm{H}_{\alpha}$ emission, the flux of the nebula measured by Pellizzoni et al. (2002) in the UKST image, where it is detected at $\gtrsim 50 \sigma$, is a factor 10 brighter than our $3 \sigma$ upper limit. This huge difference is far larger than any of the different flux calibration between the UKST and of the WFPC2 images. Thus, we exclude that the non-detection of the nebula can be attributed to an exposure shallower than expected, possibly ascribed to a decrease in the efficiency of the WFPC2 detector.

\section{Discussion}

The non-detection of the nebula in the WFPC2 $656 \mathrm{~N}$ image can be explained, in principle, by invoking a decrease in brightness since the epoch of the ESO/MPG 2.2m and of the UKST observations. However, this possibility is unlikely since the brightness measured in both ground-based observations is consistent with a steady emission. Besides, it would be difficult to explain on physical grounds such a large variability of the $\mathrm{H}_{\alpha}$ emission in both the bow-shock and the photo-ionisation nebula scenarios. It is possible that the non-detection of the nebula in the $W F P C 2656 \mathrm{~N}$ image is purely an effect of the different characteristics of the filters used in the different observations. In particular, the $\mathrm{H}_{\alpha}$ filter of the ESO/MPG 2.2m Wide Field Imager (WFI) has a central wavelength $\lambda=6588.27 \AA$ and a $F W H M$ $\Delta \lambda=74.31 \AA^{4}$, very similar to those of the $\mathrm{H}_{\alpha}$ filter of the

\footnotetext{
4 www.eso.org/sci/facilities/lasilla/instruments/wfi/ inst/filters/
} 
$\operatorname{UKST}(\lambda=6590 \AA$ and a $F W H M \Delta \lambda=70 \AA$; Parker \& Phillips 1998). Thus, both filters are centred at wavelengths longer than the rest wavelength of the $\mathrm{H}_{\alpha}$ line $(\lambda=6562.8 \AA)$ which, however, is still encompassed by their relatively large band widths. Instead, the WFPC2 $656 N$ filter $(\lambda=6564 \AA ; \Delta \lambda=22 \AA)$ is narrower and almost exactly centred on the rest wavelength of the $\mathrm{H}_{\alpha}$ line. Consequently, it is possible that the nebula is detected through the broader and redder ground-based $\mathrm{H}_{\alpha}$ filters, but not through the narrower and bluer WFPC2 656N because the emission peaks at a longer wavelength.

One possibility is that the $\mathrm{H}_{\alpha}$ line is red-shifted by $\gtrsim 10 \AA$. However, since $\mathrm{H}_{\alpha}$ emission is indeed detected in the broader ground-based filters, the line can not be red-shifted by more than $\sim 60 \AA$. This corresponds to a red-shift $0.0015 \lesssim z \lesssim 0.009$ and it would imply that the nebula is either moving or expanding asymmetrically, with a radial velocity $V_{\mathrm{r}}=450-2700 \mathrm{~km} \mathrm{~s}^{-1}$.

In the bow-shock scenario, the first case would be compatible with both the $\mathrm{CCO}$ and the shock front moving along the radial direction. This would be consistent with the shape of the nebula which features a somewhat spherical symmetry (Pellizzoni et al. 2002; Mignani et al. 2007b) instead of the more typical arc-like one. On the other hand, a nebula expanding asymmetrically would be incompatible with the fact that the shock front is expected to be almost stationary with respect to the neutron star. An asymmetric nebula expansion would also be incompatible with the photo-ionisation scenario, unless the radiation pressure from the neutron star improbably sweeps the ISM in the radial direction only. This means that a red-shifted $\mathrm{H}_{\alpha}$ line would be compatible with the bow-shock scenario only. In this case, we could constrain the CXO J085201.4-461753 rotational energy loss from its spatial velocity and from the $\mathrm{H}_{\alpha}$ luminosity of the nebula (Chatterjee \& Cordes 2002). We inferred the radial velocity $V_{\mathrm{r}}$ from the assumed red-shift of the $\mathrm{H}_{\alpha}$ line, while we derived the transverse velocity $V_{\mathrm{t}}$ from the CCO displacement from the SNR centre $\left(\approx 5^{\prime}\right)$ which, for a distance of $\sim 1 \mathrm{kpc}$ (Slane et al. 2001) and for an age of $\sim 1000-3000$ years (Katsuda et al. 2009), implies a value of $500-1500 \mathrm{~km} \mathrm{~s}^{-1}$. This corresponds to a spatial velocity $V=700-3000 \mathrm{~km} \mathrm{~s}^{-1}$, large but not unheard of for a neutron star, as it is shown by the Guitar Nebula pulsar PSR B2224+65 (see, e.g. Chatterjee \& Cordes 2002 , 2004). For an $\mathrm{H}_{\alpha}$ luminosity of $\sim 4 \times 10^{30} \mathrm{erg} \mathrm{s}^{-1}$, computed for a SNR distance of $1 \mathrm{kpc}$, and for a fraction of neutral hydrogen in the ISM $X \lesssim 1$, the bow-shock formation requires that $\dot{E} \times V \gtrsim 7 \times 10^{36}$ erg km s${ }^{-2}$ (see Fig. 3 of Pellizzoni et al. 2002). Thus, our limits on the CXO J085201.4-461753 spatial velocity translates into lower limits on its rotational energy loss of $\dot{E} \gtrsim 10^{34} \mathrm{erg} \mathrm{s}^{-1}$ and $\dot{E} \gtrsim 2 \times 10^{33} \mathrm{erg} \mathrm{s}^{-1}$, respectively. We note that the lower bound on the $\dot{E}$ of CXO J085201.4-461753 would be still compatible with the $\dot{E}$ upper limits of both CXOU J185238.6+004020 in Kes 79 (Halpern et al. 2007) and of RX J0822.0-4300 in Puppis A (Gotthelf \& Halpern 2009), and so be consistent with the emerging picture of CCOs as young, low-energetic INSs.

A second possibility is that the emission line is indeed [NII] $(\lambda=6583.6 \AA)$. In this case, the nebula would neither be produced by a velocity-driven bow-shock nor by photoionisation from the neutron star. Most likely, it would be a fore/background emission knot in the SNR or in the diffuse emission structures observed close to the CXO J085201.4-461753 position (see, e.g. Fig. 1 of Pellizzoni et al. 2002 and Fig. 2). At this stage, we can only speculate on the nebula origin. Because of its proximity, the nebula might be associated with Star Z. This possibility is also suggested by the isophotal contours of

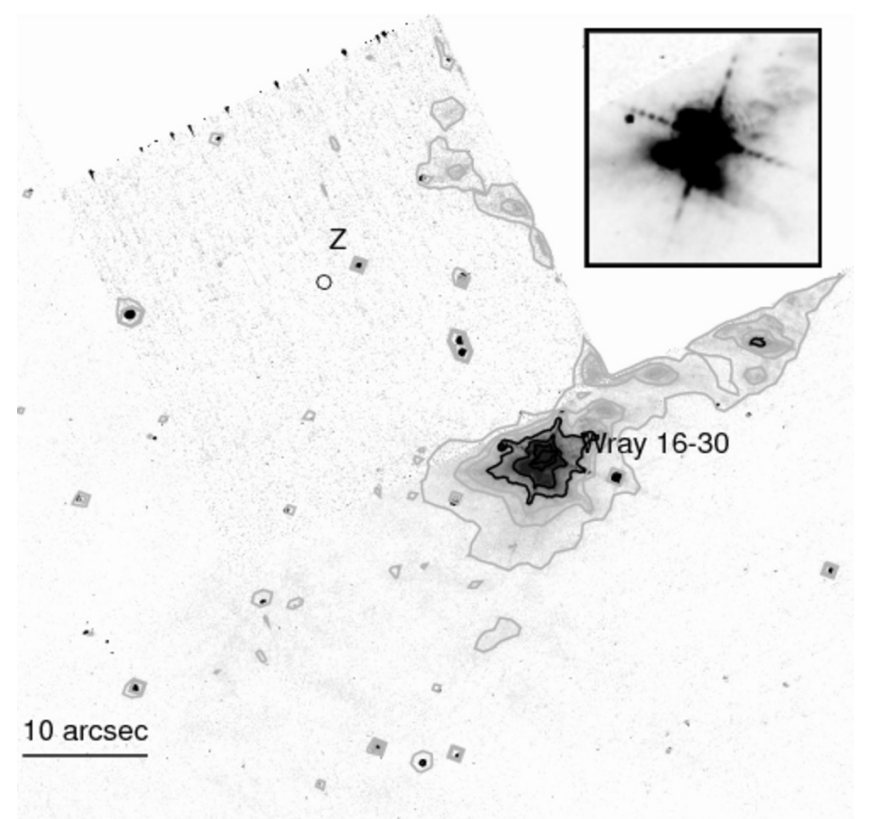

Fig. 2. $65^{\prime \prime} \times 65^{\prime \prime}$ part of the mosaic WFPC2 image around the CCO position (circle). Star Z and Wray 16-30 are labelled. North to the top, East to the left. Contours are colour-coded as in Fig. 1. Inset: $10^{\prime \prime} \times 10^{\prime \prime}$ zoom of Wray 16-30.

the UKST $\mathrm{H}_{\alpha}$ image (Fig. 1) which, within their 1" angular resolution, do not allow to exclude a physical connection between the two. The classification of Star Z is, however, uncertain. For instance, based on its $B$ and $R$-band magnitudes Pavlov et al. (2001) concluded that it might be an early G-type background star at a distance of $2.3 \mathrm{kpc}$, for an $A_{V} \sim 1.7$. Diffuse emission around main sequence stars could, in principle, be produced by gas outflows from the outer shells. However, if associated with Star Z, the nebula structure would require an unipolar outflow, difficult to explain for a main sequence star. Recently, Reynoso et al. (2006), based on 13 and $20 \mathrm{~cm}$ radio observations of the Vela Jr. SNR, suggested that star Wray 16-30 is actually a planetary nebula (PN). One could then argue whether the putative [NII] nebula seen around CXO J085201.4-461753 might be an emission knot of the external rim of the PN. However, a physical association between the two is not so evident since neither the ground-based images of Pellizzoni et al. (2002) nor our WFPC2 image (Fig. 2) show evidence of extended structures connecting Wray 16-30 to the CXO J085201.4-461753 position. We investigated the morphology of Wray 16-30 in our WFPC2 image. Thanks to the WFPC2 spatial resolution ( 0 '. 1 for the wide field camera chips), we could resolve for the first time the structure of Wray 16-30. We found that part of the object structure is indeed consistent with that of a bright, saturated, star. However, we also resolved an extended, clumpy emission structure south of it. It is not clear whether this is associated with Wray 16-30 or is part of the much more extended diffuse emission structures west/north-west of it, observed in the ground-based images and also partially visible in our WFPC2 image. Thus, from the object morphology we can not confirm the identification of Wray 16-30 as a PN.

\section{Summary}

Our HST/WFPC2 observations of the CXO J085201.4-461753 field with the $656 \mathrm{~N}$ filter, almost exactly centred on the rest 
wavelength of the $\mathrm{H}_{\alpha}$ line $(\lambda=6562.8 \AA)$, did not detect its nebula down to a $3 \sigma$ flux limit of $\sim 3 \times 10^{-15} \mathrm{erg} \mathrm{cm}^{-2} \mathrm{~s}^{-1}$, which is a factor of 10 fainter than the flux measured by Pellizzoni et al. (2002). This is probably to be ascribed to the narrower and bluer pass band of the $656 \mathrm{~N}$ filter with respect to those used in the ground-based observations (see Sect. 3), which would make the nebula undetectable if the peak of the emission is at a longer wavelength. One possibility, compatible with the bow-shock scenario only, is that the $\mathrm{H}_{\alpha}$ line is red-shifted by $\sim 10-60 \AA$ due to the neutron star motion with a radial velocity $450 \lesssim V_{\mathrm{r}} \lesssim$ $2700 \mathrm{~km} \mathrm{~s}^{-1}$. An alternative possibility is that the nebula flux is almost entirely ascribed to [NII] $(\lambda=6583.6 \AA)$ rather than to $\mathrm{H}_{\alpha}$ emission. Such a picture would then argue against its physical association with CXO J085201.4-461753. The nebula could instead be part of the complex patterns of diffuse emission that are seen in the field of CXO J085201.4-461753, possibly associated with Star Z or (at least in part) with the nearby $\left(25^{\prime \prime}\right)$ star Wray 16-30, whose nature is debated (a peculiar planetary nebula? see Reynoso et al. 2006). High-resolution, spectroscopy observations of the nebula will help to determine its nature and to establish its association with CXO J085201.4-461753.
Acknowledgements. R.P.M. thanks STFC for support through a Rolling Grant and K. Wu (UCL-MSSL) for discussions.

\section{References}

Chatterjee, S., \& Cordes, J. M. 2002, ApJ, 575, 407

Chatterjee, S., \& Cordes, J. M. 2004, ApJ, 600, L51

De Luca, A. 2008, in 40 Years of Pulsars: Millisecond Pulsars, Magnetars and More, AIP, 983, 311

Gotthelf, E. V., \& Halpern, J. P. 2007, ApJ, 664, L35

Gotthelf, E. V., \& Halpern, J. P. 2009, ApJ, 695, L35

Halpern, J. P., Gotthelf, E. V., Camilo, F., \& Seward, F. D. 2007, ApJ, 665, 1304 Katsuda, S., Tsunemi, H., \& Mori, K. 2009, ASpR, 43, 895

Lattanzi, M. G., Capetti, A., \& Macchetto, F. D. 1997, A\&A, 318, 997

Mignani, R. P., Bagnulo, S., De Luca, A., et al. 2007a, Ap\&SS, 308, 203

Mignani, R. P., de Luca, A., Zaggia, S., et al. 2007b, A\&A, 473, 883

Parker, Q. A., \& Phillips, S. 1998, PASA, 15, 28

Pavlov, G. G., Sanwal, D., Kiziltan, B., \& Garmire, G. P. 2001, ApJ, 559, L131

Pavlov, G. G., Sanwal, D., \& Teter, M. A. 2004, in Young Neutron Stars and Their Environments, ASP, 239

Pellizzoni, A., Mereghetti, S., \& De Luca, A. 2002, A\&A, 393, L65

Reynoso, E. M., Dubner, G., Giacani, E., et al. 2006, A\&A, 449,243

Skrutskie, M. F., Cutri, R. M., Stiening, et al. 2006, AJ, 131, 1163

Slane, P., Hughes, J. P., Edgar, R. J., et al. 2001, ApJ, 548, 814 